\title{
Magnetic monopoles and free fractionally charged states at accelerators and in cosmic rays
}

\author{
Thomas W. Kephart, ${ }^{a}$ George K. Leontaris ${ }^{b}$ and Qaisar Shafi ${ }^{c}$ \\ ${ }^{a}$ Department of Physics and Astronomy, Vanderbilt University, \\ Nashville, TN 37235, U.S.A. \\ ${ }^{b}$ Physics Department, Theory Division, Ioannina University, \\ GR-45110 Ioannina, Greece \\ ${ }^{c}$ Bartol Research Institute, University of Delaware, \\ Newark, DE 19716, U.S.A. \\ E-mail: tom.kephart@gmail.com, leonta@uoi.gr, shafi@bartol.udel.edu
}

ABSTRACT: Unified theories of strong, weak and electromagnetic interactions which have electric charge quantization predict the existence of topologically stable magnetic monopoles. Intermediate scale monopoles are comparable with detection energies of cosmic ray monopoles at IceCube and other cosmic ray experiments. Magnetic monopoles in some models can be significantly lighter and carry two, three or possibly even higher quanta of the Dirac magnetic charge. They could be light enough for their effects to be detected at the LHC either directly or indirectly. An example based on a D-brane inspired $\mathrm{SU}(3)_{C} \times \mathrm{SU}(3)_{L} \times \mathrm{SU}(3)_{R}$ (trinification) model with the monopole carrying three quanta of Dirac magnetic charge is presented. These theories also predict the existence of color singlet states with fractional electric charge which may be accessible at the LHC.

KEywords: Strings and branes phenomenology, Phenomenology of Field Theories in Higher Dimensions

ArXIV EPRINT: 1707.08067 


\section{Contents}

1 Introduction 1

2 Generalities for product group models 2

2.1 Pati-Salam model 2

2.2 Trinification model 2

2.3 Lowering the GUT scale 3

2.4 The 433 model 3

3 Trinification from intersecting D-brane scenario with observable monopoles

3.1 Spectrum

3.2 Gauge couplings, weak mixing angle and monopole mass in D brane trinification

4 Monopoles, inflation and primordial gravity waves 10

5 Discussion $\quad \mathbf{1 0}$

5.1 Monopoles at the LHC 11

$\begin{array}{ll}5.2 \text { Monopoles in cosmic rays } & 12\end{array}$

\section{Introduction}

The fact that electric charge is quantized lead Dirac [1] in 1931 to predict the existence of magnetic monopoles. Classically, a stationary system consisting of a magnetic monopole and an electron has a non-vanishing Poynting vector and angular momentum. Quantum mechanically, angular momentum must be quantized in units of $\hbar$ and this implies the Dirac quantization condition (in units where $\hbar=c=1$ )

$$
g q=2 n \pi,
$$

where $q$ is the electric charge, $g$ is the magnetic charge and $n$ is an integer. Dirac's argument is still compelling today but magnetic monopoles have eluded us after over eighty years of searching. The discovery of magnetic monopoles would have wide reaching implication for physics beyond the standard model. As a new energy regime has been opening up at the Large Hadron Collider ( $\mathrm{LHC}$ ), it is important to be clear on what we expect could be found as we extend the search for magnetic monopoles into this region. While some results are expected to be model dependent, others will be universal. We discuss a class of models that could have magnetic monopoles light enough to have implications for the LHC, as well as heavier monopoles that may be observed in cosmic ray experiments. 


\section{Generalities for product group models}

Two familiar examples of product gauge groups with bifundamental fermions are the PatiSalam (PS) model [2] and the trinification model [3]. In the PS model the gauge group is $\mathrm{SU}(4) \times \mathrm{SU}(2) \times \mathrm{SU}(2)$ and the fermions live in three $[(4,2,1)+(\overline{4}, 1,2)]$ families which reduces to three standard model (SM) families plus three right handed neutrinos. This model can be embedded directly into $\mathrm{SO}(10)$ with no additional fermions. In trinification models the gauge group is $\mathrm{SU}(3) \times \mathrm{SU}(3) \times \mathrm{SU}(3)$ and the fermions occupy three $[(3, \overline{3}, 1)+$ $(1,3, \overline{3})+(\overline{3}, 1,3)$ f families which reduces to three standard model (SM) families plus the additional content of three $E_{6}$ families, including additional $b$-type quarks. Let us begin by discussing the magnetic monopoles of these two models and their generalizations.

\section{$2.1 \quad$ Pati-Salam model}

It has been understood for some time that the spontaneous breaking of the Pati-Salam gauge symmetry $H=\mathrm{SU}(4)_{C} \times \mathrm{SU}(2)_{L} \times \mathrm{SU}(2)_{R}$ (422 model) yields topologically stable monopoles that carry two quanta of Dirac magnetic charge [4-6]. By not insisting that $H$ be embedded within an $\mathrm{SO}(10)$ model, this implies that in this model there should exist $\mathrm{SU}(3)_{c}$ color singlet states that carry fractional $\left( \pm \frac{e}{2}\right)$ electric charge. Adding fundamental fermions irreducible representations to the 422 model is an obvious extension. For instance, since $\mathrm{SU}(2)$ is anomaly free, and as the 422 gauge group has no U(1) factors, we could simply introduce $(1,2,1)$ and $(1,1,2)$ states in the fundamental representations of $H$ which provide the required $\mathrm{SU}(3)_{c}$ singlet states that carry fractional charge. (Recall that the known fermions belong in the bi-fundamental representations of $H$.) Moreover, we also should include the conjugate pair $(4,1,1)$ and $(\overline{4}, 1,1)$ in the fundamental representations of $H$, which transform as triplets and anti-triplets under $\mathrm{SU}(3)_{c}$ and carry fractional charge $\pm \frac{e}{6}$. These latter states could bind together to create, for instance, a new class of baryons that carry electric charge $\pm \frac{e}{2}$. They also could combine with the SM quarks to generate fractionally charged hadrons. This leads to color singlet magnetic monopoles carrying integer multiples of the Dirac charge [6], in this case $g= \pm \frac{2 e}{2 \alpha}$. In principle, the scale of the new fermions can be arranged to be light, perhaps even LHC accessible. The monopole mass depends, of course, on the 422 breaking scale. Intermediate mass monopoles may survive inflation as we will discuss.

\subsection{Trinification model}

As the gauge group for the trinification model (333 model) is $H=\mathrm{SU}(3)_{C} \times \mathrm{SU}(3)_{L} \times$ $\mathrm{SU}(3)_{R}$ and since all the $\mathrm{SU}(3) \mathrm{s}$ are potentially anomalous, the simplest generalization is to add fundamental fermion representations in conjugate pairs, e.g., $(3,1,1)+(\overline{3}, 1,1)$. There is also the possibility of adding combinations of fundamentals and bifundamentals that cancel the anomaly. For instance, we could add $[3(3,1,1)+3(1, \overline{3}, 1)]+(\overline{3}, 3,1)]$ and the theory would remain free of chiral anomalies. The additional fundamental fermions lead to leptons with electric charges $\pm \frac{2 e}{3}$ and hence charge $\pm 3 e$ magnetic monopoles. 


\subsection{Lowering the GUT scale}

If all the gauge coupling constants of a product group start off equal at the GUT scale [7, 8], then we expect the GUT scale to be rather high, $M_{U} \sim 10^{16} \mathrm{GeV}$. However, there are cases where equality at the GUT scale is not required. For instance, in orbifolded $\mathrm{AdS}_{5} / S^{5}$ with abelian orbifolding group $Z_{n}$ and gauge group $\mathrm{SU}(3)^{n}$ one finds that the gauge group coupling constants can be related by rational fractions. For trinification models the ratios are determined by how the three $\mathrm{SU}(3)$ s are diagonally embedded into the initial $\mathrm{SU}(3)^{n}$ group. (See [9] and the detailed discussion in [10].) This then allows the GUT scale to be considerably lower since less RG running is required for unification.

Another way to lower or alter the GUT scale is by adding extra dimensions to allow power law running of couplings [11-13]. Yet another is to add scalar thresholds [14-16] or vector-like fermion thresholds. All these methods can be arranged to avoid proton decay at a too rapid rate. For the remainder of this work we will assume one of these mechanisms is operating to avoid proton decay and lower the GUT scale. This will allow the GUT symmetry to break and U(1) factors to appear at a low scale, which in turn delivers light magnetic monopoles with charges depending on the gauge group and fermionic content of the model.

\subsection{The 433 model}

Now let us consider extensions of the Pati-Salam and trinification models that naturally contain both fundamental and bifundamental representations. The simplest case is based on the gauge group $\mathrm{SU}(4) \times \mathrm{SU}(3) \times \mathrm{SU}(3)$ (433 model), where both the 422 and trinification models can be embedded [17]. However, these are not the only possibilities. In all there are 18 inequivalent embeddings [18] of the standard model gauge group in $\mathrm{SU}(4) \times \mathrm{SU}(3) \times$ $\mathrm{SU}(3)$. If we insist on bifundamental fermions at the 433 level, then the 433 model is only anomaly free when families come in a multiple of three. At the 422 and trinification level, the 433 model naturally delivers both fundamental and bifundamental fermions. Hence fractional electric charge color singlets and multiply charged magnetic monopoles are natural in the 433 model.

Our main objective here is to find allowed masses and charges of magnetic monopoles and then suggest signatures for experimental searches. The 433 model is a good candidate for a model that can have detectable multicharged magnetic monopoles. (Here we focus only on models similar to the extended versions of the 422 and 333 models derivable from the 433 model and will save the exploration of the full set of non equivalently embedded SMs for further work.) The magnetic monopole spectrum for the extended versions of the 422 and 333 models under various model assumptions [19-22] then suggest where experimental searches may have the best chance of success.

\section{Trinification from intersecting D-brane scenario with observable monopoles}

In this section we explore a string motivated trinification model with monopoles that can be light enough to be observed, in future colliders as well as ongoing cosmic ray searches. 
More specifically, we will present an interesting supersymmetric version which is realised in the framework of intersecting D-branes. We will describe here the basic steps for such a viable D-brane construction. The trinification group is generated by three stacks of Dbranes, each stack containing three parallel almost coincident branes. Each stack gives rise to a $\mathrm{U}(3)$ gauge group which results in the gauge symmetry [23-25]

$$
\mathrm{U}(3)_{C} \times \mathrm{U}(3)_{L} \times \mathrm{U}(3)_{R}
$$

In this notation, the first $\mathrm{U}(3)$ contains the $\mathrm{SU}(3)$ color group of the SM gauge symmetry, the second $\mathrm{U}(3)$ includes the weak $\mathrm{SU}(2)_{L}$, and the third $\mathrm{U}(3)$ contains the $\mathrm{SU}(2)_{R}$ gauge group. From the group relation $\mathrm{U}(3) \simeq \mathrm{SU}(3) \times \mathrm{U}(1) / Z_{3}$, in addition to the standard $\mathrm{SU}(3)^{3}$ trinification gauge symmetry, the D-brane analogue is augmented by three extra U(1)'s. The final local symmetry can be written

$$
\mathrm{SU}(3)_{c} \times \mathrm{SU}(3)_{L} \times \mathrm{SU}(3)_{R} \times \mathrm{U}(1)_{C} \times \mathrm{U}(1)_{L} \times \mathrm{U}(1)_{R} .
$$

The abelian $\mathrm{U}(1)_{C, L, R}$ factors have mixed anomalies with the non-abelian $\mathrm{SU}(3)^{3}$ gauge part, but there is an anomaly free combination,

$$
\mathrm{U}(1)_{\mathcal{Z}^{\prime}}=\mathrm{U}(1)_{C}+\mathrm{U}(1)_{L}+\mathrm{U}(1)_{R}
$$

The anomalies associated with the two remaining combinations are cancelled by a generalized Green-Schwarz mechanism and the corresponding bosons receive masses from fourdimensional couplings involving the Ramond-Ramond scalars coming from the twisted closed string spectrum [26, 27]. Furthermore, there is a remaining global symmetry associated with $\mathrm{U}(1)_{C}$ of the color gauge group factor $\mathrm{U}(3)_{C} \simeq \mathrm{SU}(3)_{C} \times \mathrm{U}(1)_{C} / Z_{3}$, which can be identified with baryon number that is conserved at the perturbative level.

\subsection{Spectrum}

Next we briefly present the salient features of the spectrum. In intersecting D-branes the fermion and Higgs fields are generated by open strings with ends attached either on the same brane stack, or on two different brane stacks. In the most general picture (as in the presence of orientifolds), there are also strings with one end attached on mirror brane stacks giving rise to additional states. More precisely, open strings with ends on two different brane stacks give rise to bifundamentals, while strings with both ends on the same (or with one end on a mirror) stack introduce, among others, adjoint, antisymmetric and singlet representations. For the trinification model in particular, the bifundamentals are of the well-known form $(3, \overline{3}, 1),(1,3, \overline{3})$ and $(\overline{3}, 1,3)$.

Additional representations corresponding to open strings with ends on the same (or mirror) stacks may appear in the massless spectrum. These transform only under one gauge factor and they are formed according to $3 \times 3=\overline{3}+6$ and $3 \times \overline{3}=1+8$. Note that for the $\mathrm{SU}(3)^{3}$ symmetry, in particular, these can generate states in $(3,1,1),(1,3,1)$, and $(1,1,3)$. All of these states are 'charged' under the $\mathrm{U}(1)$ factors. 


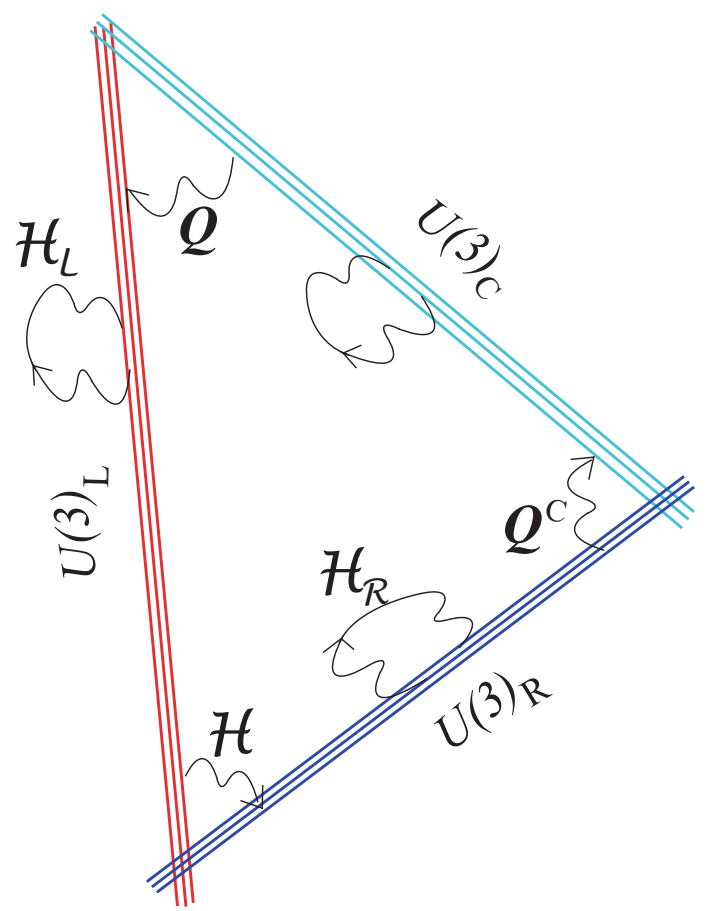

Figure 1. Intersecting D-brane stacks for the trinification model. Also shown are strings attached to D-branes whose excitations give rise to the representations explained in the text.

The standard matter representations of the 333 model arise from strings with ends on different brane stacks and have the quantum numbers

$$
\begin{aligned}
\mathcal{Q} & \left.=(3, \overline{3}, 1)_{(+1,-1,0}\right) \\
\mathcal{Q}^{c} & =(\overline{3}, 1,3)_{(-1,0,+1)} \\
\mathcal{L} & \left.=(1,3, \overline{3})_{(} \quad 0,+1,-1\right),
\end{aligned}
$$

The three lower indices refer to the three abelian factors $\mathrm{U}(1)_{C, L, R}$ discussed above. The Higgs content may be accommodated in the bifundamentals

$$
\mathcal{H}_{a}=(1,3, \overline{3})_{(0,+1,-1)}, a=1,2 .
$$

There are also representations generated with both ends on the same brane stack, such as

$$
\begin{aligned}
\mathcal{H}_{\mathcal{L}} & =(1,3,1)_{(0,-2,0)} \\
\mathcal{H}_{\mathcal{R}} & =(1,1,3)_{(0,0,-2)} \\
\mathcal{H}_{\mathcal{C}} & =(3,1,1)_{(-2,0,0)},
\end{aligned}
$$

and their complex conjugates (c.c.).

Under $\mathrm{SU}(3)_{C} \times \mathrm{SU}(2)_{L} \times \mathrm{U}(1)_{Y} \times \mathrm{U}(1)_{\Omega}\left(\right.$ where $\mathrm{U}(1)_{\Omega}$ is left over from the $\mathrm{SU}(3)_{R}$ breaking) the MSSM states have the following assignments

$$
\mathcal{Q}=q\left(3,2 ; \frac{1}{6}, 0\right)+g\left(3,1 ;-\frac{1}{3}, 0\right)
$$




$$
\begin{aligned}
\mathcal{Q}^{c}= & d^{c}\left(\overline{3}, 1 ; \frac{1}{3}, 1\right)+u^{c}\left(\overline{3}, 1 ;-\frac{2}{3}, 0\right)+g^{c}\left(\overline{3}, 1 ; \frac{1}{3},-1\right) \\
\mathcal{L}= & \ell^{+}\left(1,2 ;-\frac{1}{2}, 1\right)+\ell^{-}\left(1,2 ;-\frac{1}{2},-1\right)+\ell^{c}\left(1,2 ;+\frac{1}{2}, 0\right) \\
& +\nu^{c+}(1,1 ; 0,1)+\nu^{c-}(1,1 ; 0,-1)+e^{c}(1,1 ; 1,0),
\end{aligned}
$$

and similarly for the Higgs scalars $\mathcal{H}_{a}+$ c.c.

The 'standard' hypercharge assignment corresponds to a linear combination of the $\mathrm{U}(1)$ generators $X_{L}^{\prime}$ and $X_{R}^{\prime}$ of $\mathrm{SU}(3)_{L}$ and $\mathrm{SU}(3)_{R}$ respectively

$$
Y=-\frac{1}{6} X_{L^{\prime}}+\frac{1}{3} X_{R^{\prime}}
$$

Under the above hypercharge embedding, all MSSM particles obtained from the decompositions in (3.11) acquire their SM charges. However, we have observed that additional superfields are also available from strings with both ends attached on the same brane stack and under the hypercharge assignment (3.12), they are fractionally charged. The electric charges of the $\mathrm{SU}(2)_{L}$ triplet components $\mathcal{H}_{L}=(1,3,1)+$ c.c., in particular, are found to be fractional $\pm \frac{e}{3}, \pm \frac{2 e}{3}$.

We have seen already that in the present D-brane construction, the three additional abelian factors define the anomaly free linear combination (3.3) which can be used to redefine the hypercharge according to

$$
Y^{\prime}=Y+\frac{1}{6} \mathcal{Z}^{\prime}=-\frac{1}{6} X_{L^{\prime}}+\frac{1}{3} X_{R^{\prime}}+\frac{1}{6} \mathcal{Z}^{\prime}
$$

where $\mathcal{Z}^{\prime}$ is the generator of $\mathrm{U}(1)_{Z^{\prime}}$ in (3.3). Under this definition the hypercharge assignments of the ordinary quarks and lepton fields are not altered. In contrast, the hypercharge of the states

$$
\begin{aligned}
& \mathcal{H}_{\mathcal{L}}=(1,3,1)=\hat{h}_{L}^{+}\left(1,2 ;-\frac{1}{2}, 0\right)+\hat{\nu}_{\mathcal{H}_{\mathcal{L}}}(1,1 ; 0,0) \\
& \mathcal{H}_{\mathcal{R}}=(1,1,3)=\hat{e}_{H}^{c}(1,1 ; 1,0)+\hat{\nu}_{\mathcal{H}_{\mathcal{R}}}^{c+}(1,1 ; 0,1)+\hat{\nu}_{\mathcal{H}_{\mathcal{R}}}^{c-}(1,1 ; 0,-1),
\end{aligned}
$$

have non-zero components under $\mathrm{U}(1)_{Z^{\prime}}$ defined in (3.3), and it turns out that, with respect to (3.13), the states (3.14), (3.15) now carry the SM electric charges. Therefore, provided the $\mathrm{U}(1)_{Z^{\prime}}$ gauge boson remains massless down to the electroweak scale, the exotic fractional states do not appear in this case. The mass of $Z^{\prime}$ is affected by higher dimensional anomalies and whether it becomes massive or not, depends on the details of the particular construction.

Indeed, let $D_{a}, D_{b}$ represent two stacks of the intersecting branes, where the topology of the 6 -dimensional compact space is factorised into three tori $\mathcal{T}_{i}, i=1,2,3$. Then, the multiplicities of the chiral fermions decending from the $D_{a}-D_{b}$ bifundamentals are associated with the number of intersections

$$
I_{a b}=\prod_{i=1}^{3}\left(m_{a i} n_{b i}-m_{b i} n_{a i}\right),
$$


where $\left(n_{a i}, m_{a i}\right)$ are the winding numbers of the $D_{a}$ stack wrapping the two radii of the $i$-th torus. Similar formulae can be written for fields arising from other sectors. The restrictions on the $n_{a i}, m_{a i}$ winding numbers originating from the RR-type tadpole conditions can be readily satisfied. The mixed anomalies $\mathrm{SU}(3)_{a}^{2} \times \mathrm{U}(1)_{a}$ are proportional to $I_{a b}$ and impose additional restrictions on the $n_{a i}, m_{a i}$ sets. For instance, after dimensional reduction the ten-dimensional fields $C_{2}, C_{6}$ give the two-form fields $C_{2}=B_{0}$ and $B_{2}^{i}=\int_{\mathcal{T}_{j} \times \mathcal{T}_{k}} C_{6}$, and similar formulae hold for their duals.

The coefficients involved in the anomaly cancellation conditions depend on the winding numbers. The coefficient $c_{a}^{0}=m_{a 1} m_{a 2} m_{a 3}$, in particular, couples directly to the linear combination (3.13) through $B_{2}^{0} \wedge\left(F_{c}+F_{l}+F_{r}\right)$ where $F_{c, l, r}$ are the corresponding field strengths associated with these three U(1)'s. In general, both anomaly cancellation and fermion multiplicities require $c_{a}^{0} \neq 0$, and, as a result the corresponding gauge boson $Z^{\prime}$ becomes massive. In such a case, the new hypercharge definition cannot be implemented and so the states (3.14), (3.15) remain with exotic fractional charges.

\subsection{Gauge couplings, weak mixing angle and monopole mass in D brane trini- fication}

The various stages of the symmetry breaking chain in the D-brane trinification model are as follows. Initially, recall that for each brane stack $\mathrm{U}(3) \simeq \mathrm{SU}(3) \times \mathrm{U}(1)$. The $\mathrm{SU}(3)_{L, R}$ symmetries are assumed to break at some intermediate scale between the $Z$ boson mass $M_{Z} \approx 92 \mathrm{GeV}$ and unification scale $M_{U}$. The linear combination $\mathrm{U}(1)_{Z^{\prime}}$ may also break at any scale $M_{Z^{\prime}}<M_{U}$. However, if it is part of the hypercharge generator, this breaking should occur at low energies.

In the present trinification version the three gauge couplings $\alpha_{L, R, C}$ associated with the three sets of D-brane stacks are not necessarily equal. Hence, in principle, there is enough freedom to reconcile the low energy values of the gauge couplings with the experimental measurements. Partial unification may lead to some constraints for the intermediate breaking scales. In the most general scenario, we may assume that the gauge couplings of $\mathrm{U}(1)_{C, L, R}$ differ from those of the corresponding $\mathrm{SU}(3)$ factors (perhaps due to threshold effects, etc). Thus, we designate them with $\alpha_{L^{\prime}}, \alpha_{R^{\prime}}, \alpha_{C^{\prime}}$.

The generalized hypercharge embedding implies

$$
\frac{1}{\alpha_{Y}}=\frac{1}{3} \frac{1}{\alpha_{L}}+\frac{4}{3} \frac{1}{\alpha_{R}}+\kappa \frac{1}{6}\left(\frac{1}{\alpha_{L^{\prime}}}+\frac{1}{\alpha_{R^{\prime}}}+\frac{1}{\alpha_{C^{\prime}}}\right)
$$

where $\kappa=1$ for the general case, while for $\kappa=0$ we obtain the standard hypercharge assignment. It is convenient to define the 'harmonic' average

$$
\frac{1}{\alpha_{N}}=\frac{1}{3}\left(\frac{1}{\alpha_{L^{\prime}}}+\frac{1}{\alpha_{R^{\prime}}}+\frac{1}{\alpha_{C^{\prime}}}\right)
$$

such that

$$
\sin ^{2} \theta_{W}=\frac{3}{4\left(1+\frac{\alpha_{L}}{\alpha_{R}}\right)+\kappa \frac{3}{2} \frac{\alpha_{L}}{\alpha_{N}}}, \kappa=0,1 .
$$


For $\kappa=0$ and $\alpha_{L}=\alpha_{R}$ we obtain the standard definition and the value $\sin ^{2} \theta_{W}\left(M_{U}\right)=\frac{3}{8}$ at the GUT scale. For $\alpha_{L^{\prime}}=\alpha_{L}=\alpha_{R^{\prime}}=\alpha_{R}=\alpha_{C^{\prime}}=\alpha_{C}$ and $\kappa=1, \sin ^{2} \theta_{W}\left(M_{U}\right)=\frac{6}{19}$. For $\alpha_{L^{\prime}} \neq \alpha_{L}, \alpha_{R^{\prime}} \neq \alpha_{R}$ etc., the standard $\sin ^{2} \theta_{W}\left(M_{U}\right)=\frac{3}{8}$ is obtained if the condition $\frac{1}{\alpha_{R}}+$ $\frac{3}{8} \frac{1}{\alpha_{N}}=\frac{1}{\alpha_{L}}$ is fulfilled. Notice however, that although in a general D-brane configuration such states are possible [23, 24], in a minimal intersecting D-brane scenario with just three brane stacks, the requirement for three fermion families imply [25] a GUT mass for the gauge boson of the anomaly free $\mathrm{U}(1)_{Z^{\prime}}$ combination (3.3). In such a case this cannot be used to modify the hypercharge generator and as a result, the representations $(1,3,1)$ etc. remain with fractional electric charges. For our purposes, in search for lighter monopoles and assuming trinification breaking not too far from the EW scale, from eq. (3.17) setting $\kappa=0$, we find that $g_{R} \approx \sqrt{2} e$.

In the D-brane models a low unification scale is a plausible scenario since there is no compelling reason that the couplings unify at a high scale. As an illustrative example, let us see how this works in the present case. Let us designate the trinification scale with $M_{R}$ and define the following combination at some scale $M_{X} \leq M_{R}$ :

$$
\frac{1}{A_{X}}=\left(\frac{6}{\alpha_{Y}}-\frac{12}{\alpha_{2}}-\frac{1}{\alpha_{3}}\right)_{M_{X}} .
$$

At $M_{X}=M_{R}$, it holds $\alpha_{2}=\alpha_{L}, \alpha_{3}=\alpha_{C}$ while for the hypercharge we use formula (3.13) for $\kappa=1$ and $\alpha_{i}^{\prime}=\alpha_{i}$ (a similar analysis can be easily performed for $\kappa=0$ ). Also, for mass scales $M_{X}$ in the energy scales between $M_{R}$ and $M_{U}$, where $M_{U}$ is the GUT scale, the $\mathrm{SU}(3)_{C}$ gauge coupling is eliminated in this combination, so that

$$
\frac{1}{A_{X}}=9\left(\frac{1}{\alpha_{L}}-\frac{1}{\alpha_{R}}\right), \text { for } M_{R} \leq M_{X} \leq M_{U} .
$$

Then, the trinification breaking scale is independent of the $a_{C}$ coupling, thus the latter can be fixed independently in order to give the known low energy value for $\alpha_{3}$. We can use now the Renormalization Group Equations (RGEs) to determine the trinification breaking scale as a function of the known low energy values of the gauge couplings and beta functions. Matching the RGEs above and below the $M_{R}$ scale we find that is given by

$$
M_{R}=e^{\frac{2 \pi}{\beta-\beta^{\prime}}\left(\frac{1}{A_{Z}}-\frac{1}{A_{U}}\right)}\left(\frac{M_{U}}{M_{Z}}\right)^{\frac{\beta^{\prime}}{\beta^{\prime}-\beta}} M_{Z},
$$

where $A_{Z}$ is given by $A_{X}$ when evaluated at $M_{Z}$ and $A_{U}=A_{X}$ at $M_{X}=M_{R}$. Also, the coefficients $\beta, \beta^{\prime}$ are given by

$$
\beta=6 b_{Y}-12 b_{2}-b_{3}, \quad \beta^{\prime}=9\left(b_{R}-b_{L}\right) .
$$

For the particular case $b_{L}=b_{R}$, we get partial unification $\alpha_{L}=\alpha_{R}$ and, since then $\beta^{\prime}=0$, the scale $M_{R}$ does not depend on $M_{U}$ and is fixed only in terms of the low energy parameters. We obtain

$$
M_{R}=e^{\frac{2 \pi}{\beta} \frac{1}{A_{Z}}} M_{Z} \approx 7 \times 10^{10} \mathrm{GeV}
$$




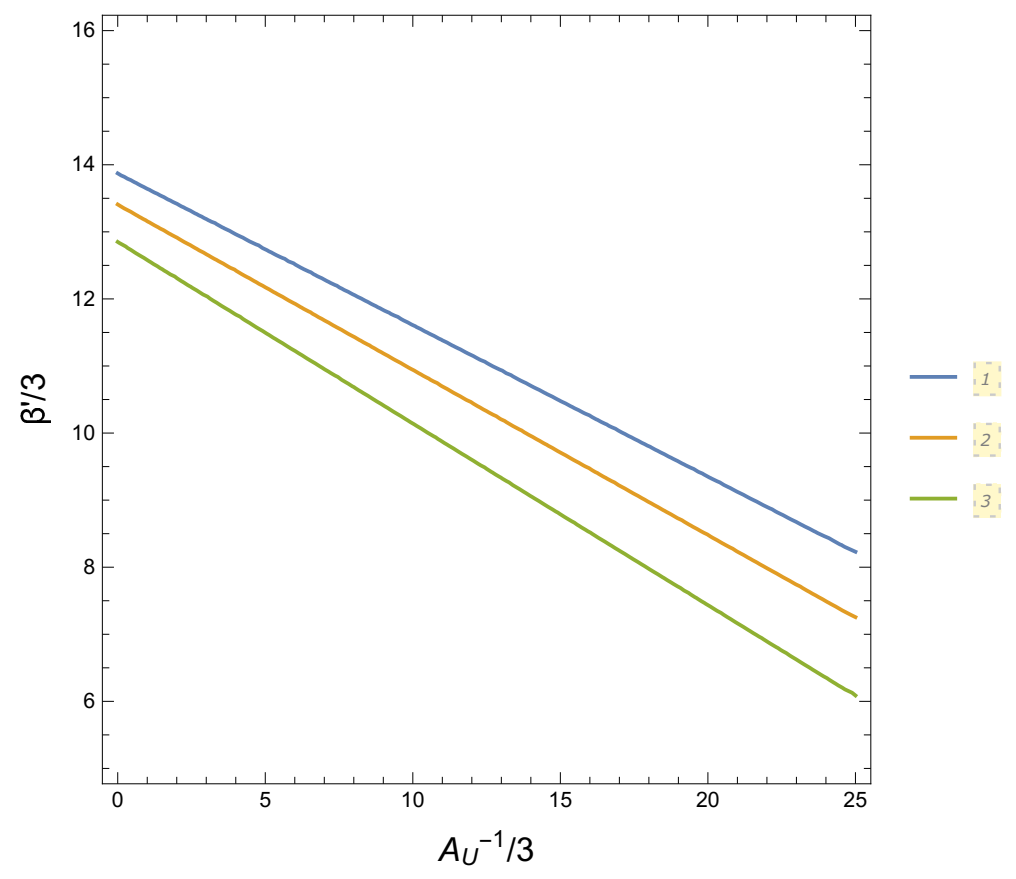

Figure 2. Contours for trinification breaking scale. The abscissa represents values for $\left(3 A_{U}\right)^{-1}$ which is proportional to the difference of the inverse gauge couplings $\alpha_{L, R}$ at the unification scale $M_{U}$ (see text). On the ordinate are the values for the difference of the corresponding beta functions $\beta^{\prime} / 3$. Curve (1) corresponds to $M_{R} \sim 10^{4} \mathrm{GeV}$, curve (2) to $M_{R} \sim 10^{5} \mathrm{GeV}$, and curve (3) to $M_{R} \sim 10^{6} \mathrm{GeV}$.

In general, however, the string boundary conditions imply $b_{L} \neq b_{R}$ and therefore various posibilities emerge. In figure (2) we show contour plots for $M_{R}=10^{4}, 10^{5}, 10^{6} \mathrm{GeV}$ in the parameter space $\frac{1}{A_{U}}, b_{R}-b_{L}$. For reasonable values $\frac{1}{\alpha_{L}, R} \sim \mathcal{O}(10)$ and $\beta^{\prime} \propto b_{R}-b_{L}$, the trinification scale can be as low as $10^{4}$ to $10^{6} \mathrm{GeV}$.

The reader might wonder whether a low trinification breaking scale could have catastrophic consequences for baryon number violating processes. Firstly, we recall that trinification symmetry does not contain gauge boson mediated dimension six proton decay operators. Secondly, as we have already pointed out, all baryon fields $\mathcal{Q}=(3, \overline{3}, 1)$ carry the same charge under the abelian symmetry $\mathrm{U}(1)_{C}$ and, therefore, the latter could play the rôle of baryon number. Finally, introducing a suitable 'matter' parity in order to distinguish the Higgs and lepton multiplets, $\mathcal{H}=\mathcal{L}=(1,3, \overline{3})$, the only allowed Yukawa coupling involving the quark fields is $\mathcal{Q} \mathcal{Q}^{c} \mathcal{H}$. Thus, proton decay can be adequately suppressed in this class of trinification models.

Before closing this section, we point out that a similar intersecting D-brane configuration can be arranged for the 422 model where states with fractional charges $\pm \frac{e}{6}, \pm \frac{e}{2}$ are generated by open strings with appropriate boundary conditions. The states with electric charges $\pm \frac{e}{6}$ also carry color and are therefore confined. 


\section{Monopoles, inflation and primordial gravity waves}

Magnetic monopoles can be problematic in the standard big bang cosmology. If they are produced at a high, $M_{U} \sim 10^{16} \mathrm{GeV}$ unification scale where a $\mathrm{U}(1)$ emerges from a non-abelian gauge group, then they overclose the Universe in the standard hot big-bang cosmology. This problem is solved by inflation which dilutes the monopoles, in some cases to levels that agree with observation. Then, it is perfectly reasonable to ask the question: how do primordial monopoles survive cosmic inflation?

This has been addressed in a number of ways by various authors and we very briefly summarize a few of them. Firstly, suppose that the spontaneous breaking of nonsupersymmetric $\mathrm{SO}(10)$ to the $\mathrm{SM}$ proceeds via the 422 symmetry, with inflation driven by an $\mathrm{SO}(10)$ singlet field [28] using the Coleman-Weinberg potential. For this case a scalar spectral index $n_{s} \sim 0.96-0.97$ is realized for a Hubble constant $H_{\text {inf }}$ during inflation of order $10^{13}-10^{14} \mathrm{GeV}$ [29]. This leads to the conclusion that monopoles associated with the breaking of 422 at an energy scale close to $H_{\text {inf }}$ can survive the inflationary epoch and be present in our galaxy at an observable level. This $\mathrm{SO}(10)$ inflationary scenario also predicts that the tensor to scalar ratio $r$, a canonical measure of gravity waves, is not much smaller than 0.02 [30], which will be tested in the near future.

A somewhat different inflationary scenario based on a quartic potential with nonminimal coupling of the inflaton field to gravity predicts an $r$ value up to an order of magnitude or so smaller [31] than the previous example. The monopole mass in this case is around $10^{13}-10^{14} \mathrm{GeV}$.

Monopoles arising in models such as supersymmetric trinification have been shown [32] to survive primordial inflation by exploiting an epoch of thermal inflation [33]-[39] which dilutes their number density to levels below the Parker bound. Depending on the model details the monopole masses can vary from the intermediate to GUT scale.

If the theory has a product group that avoids proton decay without being broken at a high scale and if the monopoles are not produced until near the electroweak scale, then they could be eliminated by late time inflation, although this may not be easy to arrange. Another possibility $[40,41]$ is to eliminate them or substantially reduce their numbers by temporarily breaking the appropriate $\mathrm{U}(1)$. Then the monopoles find themselves on the end of cosmic strings. The high tension in the strings causes efficient monopole-antimonopole annihilation thereby solving the cosmic monopole problem. Either of these mechanisms allows one to bring the monopole mass density down to a value that does not conflict with present astrophysical observations.

\section{Discussion}

Here we explore the possibility of detection of low and intermediate mass magnetic monopoles, especially those that are multiply charged. For the detection of low mass magnetic monopoles we focus on the LHC, and for the detection of intermediate mass magnetic monopoles we focus on cosmic ray experiments. 


\subsection{Monopoles at the LHC}

There have been recent suggestions of light monopoles in the standard model [19-22] and this possibility can also be explored in various branches of the 433 model. Singly charged monopoles (i.e., charge $n=1$ ) interact strongly with matter [42, 43] through their fine structure constant

$$
\alpha_{M}=\frac{1}{2 \alpha} \sim 68,
$$

and cross sections are enhanced by a factor of $n^{2}$ for multiply charged monopoles. Hence the reach of the LHC is long if it produces $M \bar{M}$ pairs. But as we discuss below, production at the LHC requires that the $M \bar{M}$ pairs are fundamental, i.e., of Dirac type, since 't Hooft-Polyakov [44, 45] monopoles, being composite, are much harder to produce and their production cross section has been estimated to be suppressed by greater than 30 orders of magnitude relative to production of fundamental point-like monopoles [46]. Hence, composite monopoles are extremely unlikely to be accessible at the LHC. (For other possibilities see also $[47,48])$

Above threshold fundamental $M \bar{M}$ pairs will be copiously produced and easily detected by their densely ionizing tracks in detectors. The MoEDAL experiments [49-54] searches for monopoles both by tracking in layered material and by monopole capture in aluminum bares that are run through superconducting detectors. Both these types of searches are carried out offline.

Monopoles will be accelerated (or decelerated) in detector magnets, and will travel on parabolic trajectories in constant magnetic fields. Hence their track will not look at all like electrically charged particles traveling on helical orbits in magnetic fields. Track reconstruction fitting routines can easily be made to distinguish the difference. Combining ionization with tracking could make a monopole track even more unmistakable.

Below threshold virtual pairs of monopoles can contribute to loop diagrams for scattering processes and alter cross sections from their predicted SM values. For example, Drell-Yan like production cross sections $q \bar{q} \rightarrow X \bar{X}$ could be enhanced.

Even though production cross sections of composite 't Hooft-Polyakov monopoles are too small for them to be produced at accelerators, this is not the case for point-like Dirac monopoles. While a full quantum theory of magnetic monopoles is lacking, limits on Dirac monopole production have been obtained via a Drell-Yan model [67], and applied to monopoles of 1, 2, 3 and 6 times the Dirac charge for $175 \mathrm{pb}^{-1}$ exposure of $p \bar{p}$ luminosity of material in the collision regions of both D0 and CDF. The resulting monopole mass limits are $256,355,410$ and $375 \mathrm{GeV} / c^{2}$ respectively, while the production cross section limits are $0.6,0.2,0.07$ and $0.2 \mathrm{pb}$ respectively.

The fractional electric charges are also very interesting in these models and potentially detectable. The electric charges are often in multiples of $\frac{1}{2} e$ or $\frac{1}{3} e$, but other fractions of $e$ are possible in certain embeddings of the SM in the 433 model. In one case particle charges come in fractions as small as $\frac{1}{12} e[18]$.

In the past, many of the best magnetic monopole limits (a comprehensive list of references can be found in the 'Magnetic Monopole Bibliography," of Giacomelli et al., [55, 56],) have been based on cosmic ray experiments [57-61], but now there is also a dedicated exper- 
iment at the LHC for this purpose. The MoEDAL experiment [49-54] mentioned above has been specifically designed to search for magnetic monopoles and other highly ionizing particles. The ATLAS experiment has also reported on their magnetic monopole search [62]. We hope the results presented here can provide additional motivation to these and other experiments.

\subsection{Monopoles in cosmic rays}

The number density of monopoles emerging from an early universe phase transition is determined by the Kibble mechanism [63,64]. From the number density we determine the flux of free monopoles with $M<10^{15} \mathrm{GeV}$ accelerated to relativistic energies by the cosmic magnetic fields. The general expression for the relativistic monopole flux may be written $[42,43]$

$$
F_{M}=c n_{M} / 4 \pi \sim 2 \times 10^{-4}\left(\frac{M}{10^{15} \mathrm{GeV}}\right)^{3}\left(\frac{l_{H}}{\xi_{c}}\right)^{3} \mathrm{~cm}^{-2} \mathrm{sec}^{-1} \mathrm{sr}^{-1}
$$

The IceCube experiment has recently put a limit on the flux of light mildly relativistic $(\beta<0.8)$ magnetic monopoles $[65,66]$

$$
\Phi_{90 \% \text { C.L. }} \sim 10^{-18} \mathrm{~cm}^{-2} \mathrm{sr}^{-1} \mathrm{~s}^{-1} .
$$

This in turn limits the cosmic density of magnetic monopoles, but it does not eliminate the possibility that cosmic monopoles were all either inflated away or annihilated at the electroweak scale but can now still be produced in accelerator or cosmic ray collisions if they are point-like particles.

Magnetic monopoles in cosmic rays could have been produced in the early universe and therefore could be of either composite 't Hooft-Polyakov type or Dirac point-like type. The Pierre Auger experiment has recently reported on a search for ultra relativistic magnetic monopoles [68] and placed limits on their flux of $1 \times 10^{-19}\left(\mathrm{~cm}^{2} \mathrm{sr} \mathrm{s}\right)^{-1}$ and $2.5 \times 10^{-21}\left(\mathrm{~cm}^{2}\right.$ $\mathrm{sr} \mathrm{s})^{-1}$ for Lorentz factors of $\gamma=10^{9}$ and $\gamma=10^{12}$ respectively, and as mentioned above, IceCube has also placed limits on the flux of relativistic and mildly relativistic magnetic monopoles [65]. For velocities above $0.51 c$ they see no flux above $1.55 \times 10^{-18}\left(\mathrm{~cm}^{2} \mathrm{sr}\right.$ $\mathrm{s})^{-1}$. The best upper limit on the flux of nonrelativistic magnetic monopoles comes from MACRO [69] who find $1.5 \times 10^{-16}\left(\mathrm{~cm}^{2} \mathrm{sr} \mathrm{s}\right)^{-1}$ for $4 \times 10^{-5}<\beta=v / c<0.5$, where all fluxes above are quoted at the $90 \%$ C.L. Numerous other experiments have also placed limits on the flux of magnetic monopoles in cosmic rays Baikal [70], SLIM [60], RICE [61] and ANITA-II [71], with the best limit on ultra relativistic magnetic monopoles coming from ANITA-II and the best limit on relativistic magnetic monopoles $\beta=0.9$ coming from IceCube [66], as discussed and summarized in [68].

\section{Acknowledgments}

Q.S. is supported in part by the DOE Grant DE-SC0013880. T.W.K and G.K.L. would like to thank the Physics and Astronomy Department and Bartol Research Institute of the University of Delaware for kind hospitality. Also, G.K.L. would like to thank LPTHE of UPMC in Paris for kind hospitality during the final stages of this work. 
Open Access. This article is distributed under the terms of the Creative Commons Attribution License (CC-BY 4.0), which permits any use, distribution and reproduction in any medium, provided the original author(s) and source are credited.

\section{References}

[1] P.A.M. Dirac, Quantized singularities in the electromagnetic field, Proc. Roy. Soc. Lond. A 133 (1931) 60 [inSPIRE].

[2] J.C. Pati and A. Salam, Lepton number as the fourth color, Phys. Rev. D 10 (1974) 275 [Erratum ibid. D 11 (1975) 703] [INSPIRE].

[3] S.L. Glashow, Trinification of all elementary particle forces, in Fifth workshop on grand unification: proceedings, K. Kang, H. Fried and P. Frampton eds., World Scientific, Singapore, (1984) [INSPIRE].

[4] G. Lazarides, M. Magg and Q. Shafi, Phase transitions and magnetic monopoles in $\mathrm{SO}(10)$, Phys. Lett. B 97 (1980) 87 [INSPIRE].

[5] Q. Shafi and C.-A. Lee, $\mathrm{SU}(4)_{C} \times \mathrm{SU}(2)_{L} \times \mathrm{SU}(2)_{R}$ models with C-parity, Phys. Lett. B 661 (2008) 33 [arXiv:0709.4637] [InSPIRE].

[6] G. Lazarides, Q. Shafi and W.P. Trower, Consequences of a monopole with Dirac magnetic charge, Phys. Rev. Lett. 49 (1982) 1756 [INSPIRE].

[7] H. Georgi, H.R. Quinn and S. Weinberg, Hierarchy of interactions in unified gauge theories, Phys. Rev. Lett. 33 (1974) 451 [INSPIRE].

[8] H. Georgi and S.L. Glashow, Unity of all elementary particle forces, Phys. Rev. Lett. 32 (1974) 438 [inSPIRE].

[9] A.E. Lawrence, N. Nekrasov and C. Vafa, On conformal field theories in four-dimensions, Nucl. Phys. B 533 (1998) 199 [hep-th/9803015] [INSPIRE].

[10] C.M. Ho, P.Q. Hung and T.W. Kephart, Conformal completion of the Standard Model with a fourth generation, JHEP 06 (2012) 045 [arXiv:1102.3997] [INSPIRE].

[11] K.R. Dienes, E. Dudas and T. Gherghetta, Extra space-time dimensions and unification, Phys. Lett. B 436 (1998) 55 [hep-ph/9803466] [INSPIRE].

[12] L.J. Hall and Y. Nomura, Gauge unification in higher dimensions, Phys. Rev. D 64 (2001) 055003 [hep-ph/0103125] [InSPIRE].

[13] G. Shiu and S.H.H. Tye, TeV scale superstring and extra dimensions, Phys. Rev. D 58 (1998) 106007 [hep-th/9805157] [INSPIRE].

[14] G.R. Dvali and Q. Shafi, Gauge hierarchy in $\mathrm{SU}(3)_{C} \times \mathrm{SU}(3)_{L} \times \mathrm{SU}(3)_{R}$ and low-energy implications, Phys. Lett. B 326 (1994) 258 [hep-ph/9401337] [INSPIRE].

[15] Z. Berezhiani, I. Gogoladze and A. Kobakhidze, TeV scale unification in four-dimensions versus extra dimensions, Phys. Lett. B 522 (2001) 107 [hep-ph/0104288] [INSPIRE].

[16] S.M. Barr and X. Calmet, Absolutely stable proton and lowering the gauge unification scale, JHEP 07 (2014) 159 [arXiv: 1404.4594] [INSPIRE].

[17] T.W. Kephart and Q. Shafi, Family unification, exotic states and magnetic monopoles, Phys. Lett. B 520 (2001) 313 [hep-ph/0105237] [INSPIRE]. 
[18] T.W. Kephart, C.-A. Lee and Q. Shafi, Family unification, exotic states and light magnetic monopoles, JHEP 01 (2007) 088 [hep-ph/0602055] [INSPIRE].

[19] Y.M. Cho and D. Maison, Monopoles in Weinberg-Salam model, Phys. Lett. B 391 (1997) 360 [hep-th/9601028] [INSPIRE].

[20] Y.M. Cho, K. Kimm and J.H. Yoon, Mass of the electroweak monopole, in Snowmass on the Mississippi Workshop (CSS2013), U.S.A., (2013) [Mod. Phys. Lett. A 31 (2016) 1650053] [arXiv:1212.3885] [INSPIRE].

[21] V. Vento and V. Sari Mantovani, On the magnetic monopole mass, arXiv:1306.4213 [INSPIRE].

[22] Y.M. Cho and J.L. Pinfold, Electroweak monopole production at the LHC - a Snowmass white paper, arXiv:1307.8390 [INSPIRE].

[23] P. Anastasopoulos, T.P.T. Dijkstra, E. Kiritsis and A.N. Schellekens, Orientifolds, hypercharge embeddings and the Standard Model, Nucl. Phys. B 759 (2006) 83 [hep-th/0605226] [INSPIRE].

[24] G.K. Leontaris and J. Rizos, $A$ D-brane inspired $\mathrm{U}(3)_{C} \times \mathrm{U}(3)_{L} \times \mathrm{U}(3)_{R}$ model, Phys. Lett. B 632 (2006) 710 [hep-ph/0510230] [INSPIRE].

[25] G.K. Leontaris, $A \mathrm{U}(3)_{C} \times \mathrm{U}(3)_{L} \times \mathrm{U}(3)_{R}$ gauge symmetry from intersecting D-branes, Int. J. Mod. Phys. A 23 (2008) 2055 [arXiv:0802.4301] [INSPIRE].

[26] L.E. Ibáñez, R. Rabadán and A.M. Uranga, Anomalous U(1)'s in type-I and type IIB D=4, $N=1$ string vacua, Nucl. Phys. B 542 (1999) 112 [hep-th/9808139] [INSPIRE].

[27] G. Aldazabal, S. Franco, L.E. Ibáñez, R. Rabadán and A.M. Uranga, D $=4$ chiral string compactifications from intersecting branes, J. Math. Phys. 42 (2001) 3103 [hep-th/0011073] [INSPIRE].

[28] G. Lazarides and Q. Shafi, Extended structures at intermediate scales in an inflationary cosmology, Phys. Lett. B 148 (1984) 35 [InSPIRE].

[29] V.N. Şenoğuz and Q. Shafi, Primordial monopoles, proton decay, gravity waves and GUT inflation, Phys. Lett. B 752 (2016) 169 [arXiv: 1510.04442] [INSPIRE].

[30] M.U. Rehman, Q. Shafi and J.R. Wickman, GUT inflation and proton decay after WMAP5, Phys. Rev. D 78 (2008) 123516 [arXiv:0810.3625] [INSPIRE].

[31] N. Okada, M.U. Rehman and Q. Shafi, Tensor to scalar ratio in non-minimal $\phi^{4}$ inflation, Phys. Rev. D 82 (2010) 043502 [arXiv: 1005.5161] [InSPIRE].

[32] G. Lazarides, C. Panagiotakopoulos and Q. Shafi, Magnetic monopoles from superstring models, Phys. Rev. Lett. 58 (1987) 1707 [INSPIRE].

[33] G. Lazarides, C. Panagiotakopoulos and Q. Shafi, Relaxing the cosmological bound on axions, Phys. Lett. B 192 (1987) 323 [INSPIRE].

[34] G. Lazarides, C. Panagiotakopoulos and Q. Shafi, Baryogenesis and the gravitino problem in superstring models, Phys. Rev. Lett. 56 (1986) 557 [INSPIRE].

[35] K. Yamamoto, Phase transition associated with intermediate gauge symmetry breaking in superstring models, Phys. Lett. B 168 (1986) 341 [INSPIRE].

[36] P. Binétruy and M.K. Gaillard, Candidates for the inflaton field in superstring models, Phys. Rev. D 34 (1986) 3069 [inSPIRE]. 
[37] G. Lazarides, C. Panagiotakopoulos and Q. Shafi, Baryogenesis in superstring motivated models, Nucl. Phys. B 307 (1988) 937 [INSPIRE].

[38] G. Lazarides and Q. Shafi, Anomalous discrete symmetries and the domain wall problem, Nucl. Phys. B 392 (1993) 61 [inSPIRE].

[39] D.H. Lyth and E.D. Stewart, Thermal inflation and the moduli problem, Phys. Rev. D 53 (1996) 1784 [hep-ph/9510204] [INSPIRE].

[40] P. Langacker and S.-Y. Pi, Magnetic monopoles in grand unified theories, Phys. Rev. Lett. 45 (1980) 1 [inSPIRE].

[41] T.H. Farris, T.W. Kephart, T.J. Weiler and T.C. Yuan, The minimal electroweak model for monopole annihilation, Phys. Rev. Lett. 68 (1992) 564 [INSPIRE].

[42] T.W. Kephart and T.J. Weiler, Magnetic monopoles as the highest energy cosmic ray primaries, Astropart. Phys. 4 (1996) 271 [astro-ph/9505134] [INSPIRE].

[43] S.D. Wick, T.W. Kephart, T.J. Weiler and P.L. Biermann, Signatures for a cosmic flux of magnetic monopoles, Astropart. Phys. 18 (2003) 663 [astro-ph/0001233] [INSPIRE].

[44] G. 't Hooft, Magnetic monopoles in unified gauge theories, Nucl. Phys. B 79 (1974) 276 [INSPIRE].

[45] A.M. Polyakov, Particle spectrum in the quantum field theory, JETP Lett. 20 (1974) 194 [Pisma Zh. Eksp. Teor. Fiz. 20 (1974) 430] [InSPIRE].

[46] A.K. Drukier and S. Nussinov, Monopole pair creation in energetic collisions: is it possible?, Phys. Rev. Lett. 49 (1982) 102 [INSPIRE].

[47] Ya. B. Zeldovich and M. Yu. Khlopov, On the concentration of relic magnetic monopoles in the universe, Phys. Lett. B 79 (1978) 239 [InSPIRE].

[48] D. Stojkovic, G.D. Starkman and R. Matsuo, Dark energy, the colored anti-de Sitter vacuum and LHC phenomenology, Phys. Rev. D 77 (2008) 063006 [hep-ph/0703246] [INSPIRE].

[49] MoEDAL collaboration, J. Pinfold, MoEDAL becomes the LHC's magnificent seventh, CERN Cour. 50N4 (2010) 19 [INSPIRE].

[50] MoEDAL collaboration, B. Acharya et al., Search for magnetic monopoles with the MoEDAL prototype trapping detector in $8 \mathrm{TeV}$ proton-proton collisions at the $\mathrm{LHC}$, JHEP 08 (2016) 067 [arXiv: 1604.06645] [INSPIRE].

[51] MoEDAL collaboration, M. King, Simulation of the MoEDAL experiment, Nucl. Part. Phys. Proc. 273-275 (2016) 2560 [InSPIRE].

[52] MoEDAL collaboration, J.L. Pinfold, The MoEDAL experiment at the LHC. Searching beyond the Standard Model, EPJ Web Conf. 126 (2016) 02024 [INSPIRE].

[53] MoEDAL collaboration, B. Acharya et al., Search for magnetic monopoles with the MoEDAL forward trapping detector in $13 \mathrm{TeV}$ proton-proton collisions at the LHC, Phys. Rev. Lett. 118 (2017) 061801 [arXiv:1611.06817] [INSPIRE].

[54] MoEDAL collaboration, N.E. Mavromatos and V.A. Mitsou, Physics reach of MoEDAL at LHC: magnetic monopoles, supersymmetry and beyond, arXiv:1612.07012 [INSPIRE].

[55] G. Giacomelli et al., Magnetic monopole bibliography, hep-ex/0005041 [INSPIRE].

[56] S. Balestra et al., Magnetic monopole bibliography - II, arXiv:1105.5587 [INSPIRE]. 
[57] B.J. Christy, A search for relativistic magnetic monopoles with the IceCube 22-string detector, Ph.D. dissertation, Maryland U., College Park U.S.A., (2011) [InSPIRE].

[58] ANTARES collaboration, S. Adrian-Martinez et al., Search for relativistic magnetic monopoles with the ANTARES neutrino telescope, Astropart. Phys. 35 (2012) 634 [arXiv:1110.2656] [INSPIRE].

[59] KM3NET collaboration, V. Popa, KM3NeT: present status and potentiality for the search for exotic particles, Nucl. Instrum. Meth. A 630 (2011) 125 [INSPIRE].

[60] S. Balestra et al., Magnetic monopole search at high altitude with the SLIM experiment, Eur. Phys. J. C 55 (2008) 57 [arXiv:0801.4913] [INSPIRE].

[61] D.P. Hogan, D.Z. Besson, J.P. Ralston, I. Kravchenko and D. Seckel, Relativistic magnetic monopole flux constraints from RICE, Phys. Rev. D 78 (2008) 075031 [arXiv:0806.2129] [INSPIRE].

[62] ATLAS collaboration, Search for magnetic monopoles in $\sqrt{s}=7 \mathrm{TeV}$ pp collisions with the ATLAS detector, ATLAS-CONF-2012-062, CERN, Geneva Switzerland, (2012).

[63] T.W.B. Kibble, Topology of cosmic domains and strings, J. Phys. A 9 (1976) 1387 [INSPIRE].

[64] T.W.B. Kibble, Some implications of a cosmological phase transition, Phys. Rept. 67 (1980) 183 [INSPIRE].

[65] ICECube collaboration, M.G. Aartsen et al., Searches for relativistic magnetic monopoles in IceCube, Eur. Phys. J. C 76 (2016) 133 [arXiv:1511.01350] [INSPIRE].

[66] ICECube collaboration, R. Abbasi et al., Search for relativistic magnetic monopoles with IceCube, Phys. Rev. D 87 (2013) 022001 [arXiv:1208.4861] [INSPIRE].

[67] G.R. Kalbfleisch, W. Luo, K.A. Milton, E.H. Smith and M.G. Strauss, Limits on production of magnetic monopoles utilizing samples from the D0 and CDF detectors at the Tevatron, Phys. Rev. D 69 (2004) 052002 [hep-ex/0306045] [INSPIRE].

[68] Pierre Auger collaboration, A. Aab et al., Search for ultrarelativistic magnetic monopoles with the Pierre Auger Observatory, Phys. Rev. D 94 (2016) 082002 [arXiv:1609.04451] [INSPIRE].

[69] MACRO collaboration, M. Ambrosio et al., Final results of magnetic monopole searches with the MACRO experiment, Eur. Phys. J. C 25 (2002) 511 [hep-ex/0207020] [INSPIRE].

[70] Baikal collaboration, V. Aynutdinov et al., Search for relativistic magnetic monopoles with the Baikal Neutrino Telescope NT200, astro-ph/0507713 [INSPIRE].

[71] ANITA-II collaboration, M. Detrixhe et al., Ultra-relativistic magnetic monopole search with the ANITA-II balloon-borne radio interferometer, Phys. Rev. D 83 (2011) 023513 [arXiv: 1008.1282] [INSPIRE]. 